Supplement of Biogeosciences, 12, 757-767, 2015

http://www.biogeosciences.net/12/757/2015/

doi:10.5194/bg-12-757-2015-supplement

(C) Author(s) 2015. CC Attribution 3.0 License.

(c) (i)

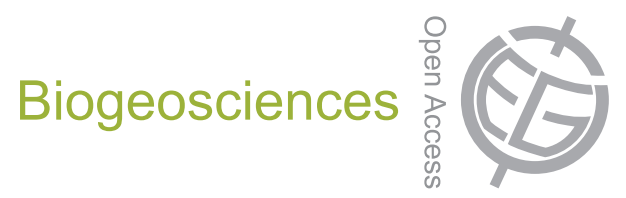

Supplement of

\title{
Strong stoichiometric resilience after litter manipulation experiments; a case study in a Chinese grassland
}

\section{Xiao et al.}

Correspondence to: B. Guenet (bertrand.guenet@1sce.ipsl.fr) and C. Xiao (cwxiao@ibcas.ac.cn) 


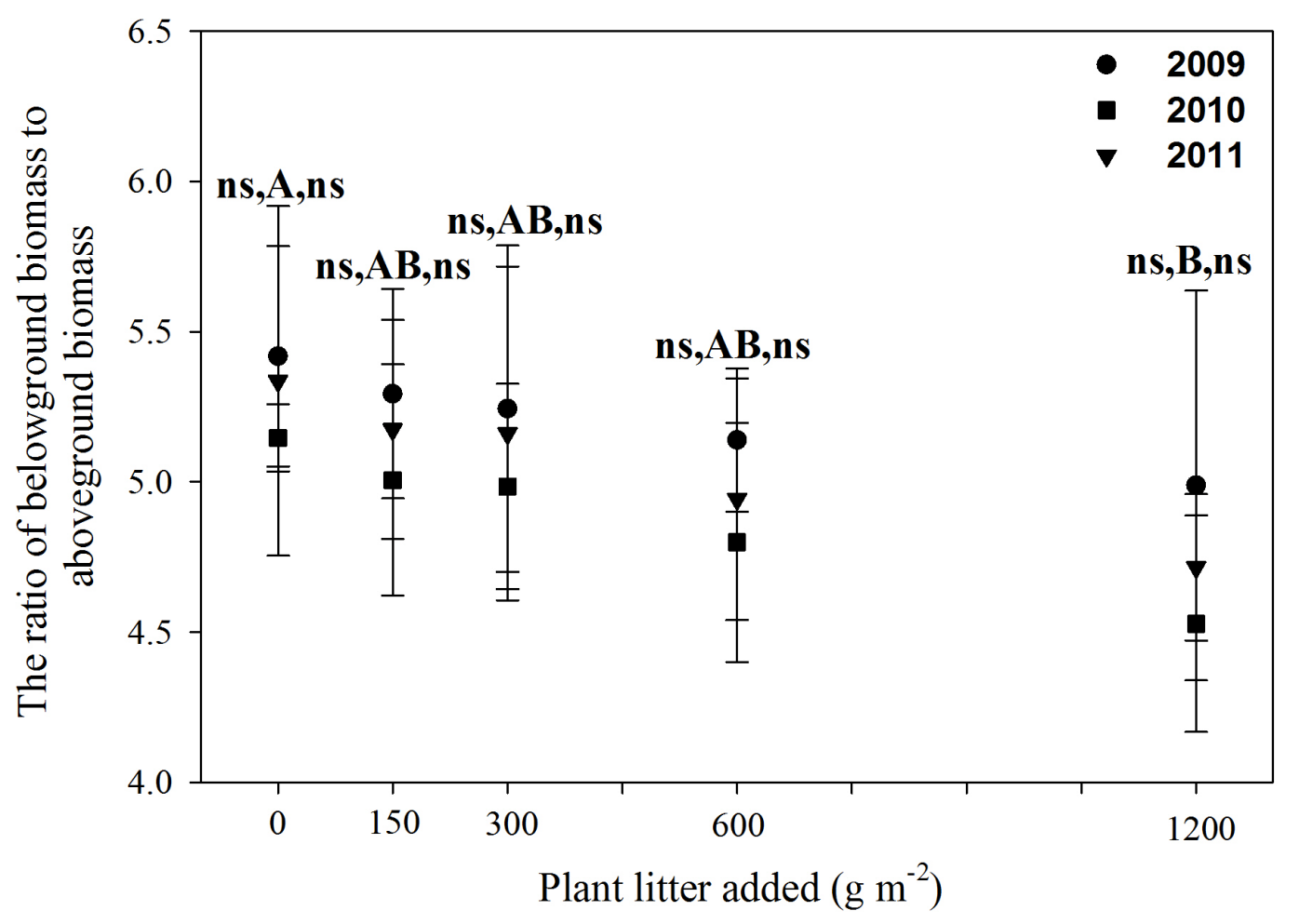

3

4 Fig. S1 Ratio of belowground biomass to aboveground biomass in 2009, 2010 and 2011

5 under different amounts of litter addition in a steppe community of northern China. Vertical

6 bars indicate one standard error about the mean $(n=5)$. A significant year effect $(p<0.05)$ was

7 detected but no interaction between litter addition and year. Treatments with different letters

8 are significantly different $(P<0.05)$ according to the Duncan test, small letters, capital letters

9 and Greek letters correspond to the year 2009, 2010 and 2011, respectively. 'ns' implies that

10 no significant differences were detected. 
Table S1: Linear regression equations for each variables

\begin{tabular}{|c|c|c|c|c|}
\hline $\begin{array}{l}\text { Corresponding } \\
\text { figures }\end{array}$ & Year & Linear regression equations & $\mathrm{R}^{2}$ & $p$ \\
\hline \multirow{3}{*}{$\begin{array}{l}\text { Figure } 2(\mathrm{~A}), \\
\text { inorganic N }\end{array}$} & 2009 & $y=0.0003 x+2.2789$ & 0.9880 & 0.0006 \\
\hline & 2010 & $y=0.0004 x+2.3823$ & 0.9223 & 0.0094 \\
\hline & 2011 & $y=0.0004 x+2.2678$ & 0.9956 & 0.0001 \\
\hline \multirow{3}{*}{$\begin{array}{l}\text { Figure } 2(\mathrm{~B}), \\
\text { available } \mathrm{P}\end{array}$} & 2009 & $y=5.45^{*} 10^{-5} x+0.7119$ & 0.9904 & 0.0004 \\
\hline & 2010 & $y=9.93 * 10^{-5} x+0.7350$ & 0.9059 & 0.0126 \\
\hline & 2011 & $y=7.77 * 10^{-5} x+0.7113$ & 0.9940 & 0.0002 \\
\hline \multirow{3}{*}{$\begin{array}{c}\text { Figure } 3(\mathrm{~A}), \\
\text { aboveground biomass }\end{array}$} & 2009 & $y=0.0250 x+187.7169$ & 0.9581 & 0.0037 \\
\hline & 2010 & $y=0.0434 x+200.2773$ & 0.9711 & 0.0021 \\
\hline & 2011 & $y=0.0369 x+194.1881$ & 0.9429 & 0.0038 \\
\hline \multirow{3}{*}{$\begin{array}{c}\text { Figure } 3(\mathrm{~B}), \\
\text { belowground } \\
\text { biomass }\end{array}$} & 2009 & $y=0.0631 x+1002.84$ & 0.9265 & 0.0086 \\
\hline & 2010 & $y=0.0946 x+1025.49$ & 0.8681 & 0.0212 \\
\hline & 2011 & $y=0.0836 x+1021.35$ & 0.8641 & 0.0222 \\
\hline \multirow{3}{*}{$\begin{array}{c}\text { Figure } 3(\mathrm{C}), \text { total } \\
\text { biomass }\end{array}$} & 2009 & $y=0.0882 x+1190.56$ & 0.9373 & 0.0068 \\
\hline & 2010 & $y=0.1380 x+1225.77$ & 0.9073 & 0.0123 \\
\hline & 2011 & $y=0.1205 x+1215.46$ & 0.8979 & 0.0143 \\
\hline \multirow{3}{*}{ Figure 3 (D), litter } & 2009 & $y=0.0070 x+42.85$ & 0.9247 & 0.0090 \\
\hline & 2010 & $y=0.0097 x+46.11$ & 0.8676 & 0.0213 \\
\hline & 2011 & $y=0.0077 x+43.72$ & 0.8719 & 0.0203 \\
\hline \multirow{3}{*}{$\begin{array}{c}\text { Figure } 4(\mathrm{~A}), \mathrm{C} \text { in } \\
\text { aboveground biomass }\end{array}$} & 2009 & $y=0.0107 x+79.2106$ & 0.9750 & 0.0017 \\
\hline & 2010 & $y=0.0177 x+85.0269$ & 0.9652 & 0.0028 \\
\hline & 2011 & $y=0.0146 x+82.6590$ & 0.9480 & 0.0051 \\
\hline \multirow{3}{*}{$\begin{array}{c}\text { Figure } 4(\mathrm{~B}), \mathrm{N} \text { in } \\
\text { aboveground biomass }\end{array}$} & 2009 & $y=0.0005 x+2.7111$ & 0.9757 & 0.0016 \\
\hline & 2010 & $y=0.0010 x+2.9748$ & 0.9676 & 0.0025 \\
\hline & 2011 & $y=0.0008 x+2.8192$ & 0.9759 & 0.0016 \\
\hline \multirow{3}{*}{$\begin{array}{c}\text { Figure } 4(\mathrm{C}), \mathrm{P} \text { in } \\
\text { aboveground biomass }\end{array}$} & 2009 & $y=3.92 * 10^{-5} x+0.1993$ & 0.9561 & 0.0046 \\
\hline & 2010 & $y=6.83 * 10^{-5} x+0.2164$ & 0.9692 & 0.0023 \\
\hline & 2011 & $y=5.90 * 10^{-5} x+0.2059$ & 0.9619 & 0.0032 \\
\hline \multirow{3}{*}{$\begin{array}{c}\text { Figure } 4(\mathrm{D}), \mathrm{C} \text { in } \\
\text { belowground } \\
\text { biomass }\end{array}$} & 2009 & $y=0.0212 x+410.76$ & 0.9627 & 0.0031 \\
\hline & 2010 & $y=0.0286 x+418.53$ & 0.9081 & 0.0122 \\
\hline & 2011 & $y=0.0367 x+414.64$ & 0.8450 & 0.0272 \\
\hline \multirow{2}{*}{$\begin{array}{l}\text { Figure } 4(\mathrm{E}), \mathrm{N} \text { in } \\
\text { belowground }\end{array}$} & 2009 & $y=0.0013 x+114.291$ & 0.9675 & 0.0025 \\
\hline & 2010 & $y=0.0018 x+11.7406$ & 0.9144 & 0.0109 \\
\hline
\end{tabular}




\begin{tabular}{|c|c|c|c|c|}
\hline biomass & 2011 & $y=0.0016 x+11.6726$ & 0.9159 & 0.0106 \\
\hline \multirow{3}{*}{$\begin{array}{l}\text { Figure } 4(\mathrm{~F}), \mathrm{P} \text { in } \\
\text { belowground } \\
\text { biomass }\end{array}$} & 2009 & $y=0.0001 x+0.9669$ & 0.9485 & 0.0050 \\
\hline & 2010 & $y=0.0002 x+0.9956$ & 0.9475 & 0.0052 \\
\hline & 2011 & $y=0.0001 x+0.9878$ & 0.9279 & 0.0084 \\
\hline \multirow{3}{*}{$\begin{array}{c}\text { Figure } 4(\mathrm{G}), \mathrm{C} \text { in } \\
\text { litter }\end{array}$} & 2009 & $y=0.0029 x+17.3641$ & 0.8969 & 0.0145 \\
\hline & 2010 & $y=0.0041 x+18.6355$ & 0.8472 & 0.0266 \\
\hline & 2011 & $y=0.0033 x+17.7622$ & 0.8657 & 0.0218 \\
\hline \multirow{3}{*}{$\begin{array}{c}\text { Figure } 4(\mathrm{H}), \mathrm{N} \text { in } \\
\text { litter }\end{array}$} & 2009 & $\mathrm{y}=9.63 * 10^{-5} \mathrm{x}+0.4240$ & 0.9283 & 0.0083 \\
\hline & 2010 & $y=0.0001 x+0.4580$ & 0.9384 & 0.0065 \\
\hline & 2011 & $y=0.0001 x+0.4353$ & 0.9071 & 0.0124 \\
\hline \multirow{3}{*}{$\begin{array}{c}\text { Figure } 4(\mathrm{I}), \mathrm{P} \text { in } \\
\text { litter }\end{array}$} & 2009 & $\mathrm{y}=7.57 * 10^{-6} \mathrm{x}+0.0345$ & 0.9304 & 0.0080 \\
\hline & 2010 & $\mathrm{y}=1.14 * 10^{-5} \mathrm{x}+0.0375$ & 0.8822 & 0.0178 \\
\hline & 2011 & $\mathrm{y}=8.99 * 10^{-6} \mathrm{x}+0.0353$ & 0.8755 & 0.0194 \\
\hline \multirow[t]{3}{*}{ Figure $4(\mathrm{~J}), \mathrm{C}$ in soil } & 2009 & $y=0.2489 x+4041.38$ & 0.9992 & $<0.0001$ \\
\hline & 2010 & $y=0.2642 x+4052.44$ & 0.9952 & 0.0001 \\
\hline & 2011 & $y=0.2529 x+4047.41$ & 0.9992 & $<0.0001$ \\
\hline \multirow{3}{*}{$\begin{array}{c}\text { Figure } 4(\mathrm{~K}), \mathrm{N} \text { in } \\
\text { soil }\end{array}$} & 2009 & $y=0.0077 x+451.71$ & 0.9975 & $<0.0001$ \\
\hline & 2010 & $y=0.0067 x+449.47$ & 0.9839 & 0.0009 \\
\hline & 2011 & $y=0.0071 x+452.25$ & 0.9902 & 0.0004 \\
\hline \multirow[t]{3}{*}{ Figure 4 (L), $P$ in soil } & 2009 & $y=0.0006 x+75.6024$ & 0.9911 & 0.0004 \\
\hline & 2010 & $y=0.0005 x+75.3127$ & 0.9684 & 0.0024 \\
\hline & 2011 & $y=0.0005 x+75.5776$ & 0.9789 & 0.0013 \\
\hline \multirow{3}{*}{$\begin{array}{l}\text { Figure } 4(\mathrm{M}), \mathrm{C} \text { in } \\
\text { soil microbial } \\
\text { biomass }\end{array}$} & 2009 & $y=0.0117 x+50.0637$ & 0.8796 & 0.0184 \\
\hline & 2010 & $y=0.0136 x+53.2399$ & 0.9231 & 0.0093 \\
\hline & 2011 & $y=0.0113 x+51.2838$ & 0.9290 & 0.0082 \\
\hline \multirow{3}{*}{$\begin{array}{l}\text { Figure } 4(\mathrm{~N}), \mathrm{N} \text { in } \\
\text { soil microbial } \\
\text { biomass }\end{array}$} & 2009 & $y=0.0020 x+4.1233$ & 0.9957 & 0.0001 \\
\hline & 2010 & $y=0.0027 x+4.7870$ & 0.9952 & 0.0001 \\
\hline & 2011 & $y=0.0023 x+4.3665$ & 0.9969 & $<0.0001$ \\
\hline \multirow{3}{*}{$\begin{array}{c}\text { Figure } 5(\mathrm{~A}), \mathrm{C}: \mathrm{N} \\
\text { ratio in aboveground } \\
\text { biomass }\end{array}$} & 2009 & $y=-0.0010 x+29.2102$ & 0.9606 & 0.0034 \\
\hline & 2010 & $y=-0.0025 x+28.6053$ & 0.9332 & 0.0075 \\
\hline & 2011 & $y=-0.0023 x+29.3773$ & 0.9927 & 0.0003 \\
\hline \multirow{3}{*}{$\begin{array}{l}\text { Figure } 5(\mathrm{~B}), \mathrm{C}: \mathrm{P} \\
\text { ratio in aboveground } \\
\text { biomass }\end{array}$} & 2009 & $y=-0.0218 x+398.67$ & 0.8214 & 0.0339 \\
\hline & 2010 & $y=-0.0319 x+393.06$ & 0.9556 & 0.0040 \\
\hline & 2011 & $y=-0.0325 x+400.83$ & 0.9626 & 0.0031 \\
\hline Figure $5(\mathrm{C}), \mathrm{N}: \mathrm{P}$ & 2009 & NS & & \\
\hline
\end{tabular}




\begin{tabular}{|c|c|c|c|c|}
\hline \multirow{2}{*}{$\begin{array}{l}\text { ratio in aboveground } \\
\text { biomass }\end{array}$} & 2010 & NS & & \\
\hline & 2011 & NS & & \\
\hline \multirow{3}{*}{$\begin{array}{c}\text { Figure } 5(\mathrm{D}), \mathrm{C}: \mathrm{N} \\
\text { ratio in belowground } \\
\text { biomass }\end{array}$} & 2009 & $y=-0.0020 x+36.0898$ & 0.9327 & 0.0076 \\
\hline & 2010 & $y=-0.0019 x+35.7446$ & 0.8531 & 0.0250 \\
\hline & 2011 & $y=-0.0014 x+35.6171$ & 0.9793 & 0.0013 \\
\hline \multirow{3}{*}{$\begin{array}{c}\text { Figure } 5(\mathrm{E}), \mathrm{C}: \mathrm{P} \\
\text { ratio in belowground } \\
\text { biomass }\end{array}$} & 2009 & $y=-0.0250 x+426.41$ & 08917 & 0.0157 \\
\hline & 2010 & $y=-0.0276 x+421.92$ & 0.9586 & 0.0036 \\
\hline & 2011 & $y=-0.0238 x+422.69$ & 0.9435 & 0.0058 \\
\hline \multirow{3}{*}{$\begin{array}{c}\text { Figure } 5(\mathrm{~F}), \mathrm{N}: \mathrm{P} \\
\text { ratio in belowground } \\
\text { biomass }\end{array}$} & 2009 & NS & & \\
\hline & 2010 & NS & & \\
\hline & 2011 & $y=-0.0002 x+11.9062$ & 0.8075 & 0.0382 \\
\hline \multirow{3}{*}{$\begin{array}{l}\text { Figure } 5(\mathrm{G}), \mathrm{C}: \mathrm{N} \\
\quad \text { ratio in litter }\end{array}$} & 2009 & $y=-0.0019 x+40.9642$ & 0.8610 & 0.0230 \\
\hline & 2010 & $y=-0.0030 x+40.6665$ & 0.9893 & 0.0005 \\
\hline & 2011 & $y=-0.0024 x+40.8086$ & 0.9662 & 0.0027 \\
\hline \multirow{3}{*}{$\begin{array}{l}\text { Figure } 5(\mathrm{H}) \mathrm{C}: \mathrm{P} \\
\quad \text { ratio in litter }\end{array}$} & 2009 & $y=-0.0240 x+505.72$ & 0.8982 & 0.0142 \\
\hline & 2010 & $y=-0.0317 x+497.57$ & 0.9324 & 0.0076 \\
\hline & 2011 & $y=-0.0290 x+503.36$ & 0.79937 & 0.040 \\
\hline \multirow{3}{*}{$\begin{array}{c}\text { Figure } 5 \text { (I), N:P ratio } \\
\text { in litter }\end{array}$} & 2009 & NS & & \\
\hline & 2010 & NS & & \\
\hline & 2011 & NS & & \\
\hline \multirow{3}{*}{$\begin{array}{l}\text { Figure } 5(\mathrm{~J}), \mathrm{C}: \mathrm{N} \\
\text { ratio in soil }\end{array}$} & 2009 & $y=0.0004 x+8.9602$ & 0.9954 & 0.0001 \\
\hline & 2010 & $y=0.0004 x+9.0321$ & 0.9844 & 0.0008 \\
\hline & 2011 & $y=0.0004 x+8.9652$ & 0.9960 & 0.0001 \\
\hline \multirow{3}{*}{$\begin{array}{l}\text { Figure } 5(\mathrm{~K}), \mathrm{C}: \mathrm{P} \\
\text { ratio in soil }\end{array}$} & 2009 & $y=0.0029 x+53.5717$ & 0.9986 & 0.0001 \\
\hline & 2010 & $y=0.0032 x+53.9324$ & 0.9911 & 0.0004 \\
\hline & 2011 & $y=0.0030 x+53.6731$ & 0.9984 & $<0.0001$ \\
\hline \multirow{3}{*}{$\begin{array}{l}\text { Figure } 5(\mathrm{~L}), \mathrm{N}: \mathrm{P} \\
\text { ratio in soil }\end{array}$} & 2009 & $\mathrm{y}=5.42 * 10^{-5} \mathrm{x}+5.9787$ & 0.9851 & 0.0008 \\
\hline & 2010 & $y=5.17 * 10^{-5} x+5.9717$ & 0.9298 & 0.0081 \\
\hline & 2011 & $y=5.55^{*} 10^{-5} x+5.9868$ & 0.9293 & 0.0081 \\
\hline \multirow{3}{*}{$\begin{array}{l}\text { Figure } 5(\mathrm{M}), \text { ), C:N } \\
\text { ratio in soil microbial } \\
\text { biomass }\end{array}$} & 2009 & $y=-0.0019 x+12.0448$ & 0.9420 & 0.0060 \\
\hline & 2010 & $y=-0.0021 x+11.0743$ & 0.9899 & 0.0004 \\
\hline & 2011 & $y=-0.0022 x+11.6713$ & 0.9703 & 0.0022 \\
\hline \multirow{3}{*}{$\begin{array}{c}\text { Supplementary } \\
\text { Figure 1, } \\
\text { belowground } \\
\text { biomass:aboveground } \\
\text { biomass ratio }\end{array}$} & 2009 & $y=-0.0003 x+5.3669$ & 0.9530 & 0.0044 \\
\hline & 2010 & $y=-0.0005 x+5.2911$ & 0.9695 & 0.0023 \\
\hline & 2011 & $y=-0.0005 x+5.1179$ & 0.9872 & 0.0006 \\
\hline
\end{tabular}


